\title{
Analytical solution on transient aerodynamic heating of hypersonic vehicle hot structure
}

\author{
Qin Lu ${ }^{1}$, Ping Zhang ${ }^{2, a}$, Longfei Hu ${ }^{1}$ and Guiqing Jiang ${ }^{1}$ \\ ${ }^{1}$ China Academy of Aerospace Aerodynamics, Beijing 100074,China \\ ${ }^{2}$ Beijing Institute Aerospace Test Technology, Beijing 100074,China
}

\begin{abstract}
New type hypersonic vehicles become more and more important due to its high performance, safety and low price. Thermal protection system(TPS), Which are correlated to the safety of hypersonic vehicles, are one of the most critical techniques in design and manufacture of hypersonic vehicles. The reusable ceramic tiles, such as $\mathrm{C} / \mathrm{C}, \mathrm{C} / \mathrm{SiC}$ and insulator tiles would be susceptible to excessive recession during retry and fatal weakness of UHTCs is prone to thermal fragmentation. An analytical solution of a simplified transient thermal problem was derived for thermal response on non-ablation materials subjected to a simplified heat plus. The solution is introduced two non-dimensional parameters. Analysis of influencing factors on transient thermal response has been performed. Results and data obtained from calculation will gie guidance to thermal protection design.
\end{abstract}

Keywords: hot structure; thermal response; high temperature; analytical solution.

\section{Introduction}

New type hypersonic vehicles become more and more important due to its high performance, safety and low price. Thermal protection system (TPS), Which are correlated to the safety of hypersonic vehicles, are one of the most critical techniques in design and manufacture of hypersonic vehicles.

New-type hypersonic vehicle will encounter significant challenges due to severe thermal heating environments during its long-time hypersonic flying in the atmosphere. Traditional hypersonic vehicles are of high enthalpy, high heat flux and short time reentry, while new type hypersonic vehicles with high enthalpy, non-high heat flux and long time reentry.Four main requirements for TPS have been put forwards for new hypersonic vehicles: high temperature capability, high efficient insulator, lightweight and low cost, etc. However, traditional ablation TPS doesn't meet the new requirements. There are some problems on non-ablation thermal protection materials: The reusable ceramic tiles, such as $\mathrm{C} / \mathrm{C}, \mathrm{C} / \mathrm{SiC}$ and insulator tiles would be susceptible to excessive recession during re-entry. Therefore, it is necessary to perform analysis and sizing for transient aerodynamic heating of hot structure in order to give guidance to thermal protection design.

${ }^{a}$ Corresponding author : luqinbj@sina.cn 


\section{Impact factor analysis on transient aerodynamic heating}

The ultimate aim of TPS is to ensure the safety and operation smoothly of whole vehicle. Temperature, thermal stress, weight and so on are some important parameters of TPS design which are affected by thermal environment and physical parameters.

Nowadays, many researches focusing thermal protection materials have been done. Numerical computations have been used for transient aerodynamic heating, while few researches focusing on analytical solution have been done. A simplified transient thermal problem was investigated in an attempt to gain basic insight into thermal response of non-ablation materials subjected to a transient aerodynamic heating plus. An analytical solution of the transient thermal problem was derived for thermal response on non-ablation materials subjected to a simplified heat plus. The solution is introduced two non-dimensional parameters. These two variables account for the thermal material properties as well as for the geometric parameters of structure and combine thermal material properties with thermal environment parameters. Analysis of influencing factors on transient thermal response has been performed.

\section{Non-dimension transient heat conduction equations}

When the flow velocity across the vehicles is not fast, and temperature is not enough high, the aerodynamic heating can be written as:

$$
\mathrm{q}_{\mathrm{w}}=\mathrm{h}\left(\mathrm{T}_{\mathrm{r}}-\mathrm{T}_{\mathrm{w}}\right)
$$

Where, $\mathrm{q}_{\mathrm{w}}$ is wall heat flux, $\mathrm{T}_{\mathrm{r}}$ is adiabatic temperature, $\mathrm{T}_{\mathrm{w}}$ is wall temperature and $\mathrm{h}$ is convection coefficient.

In above case, for an infinite plate with thickness $\delta$, one dimension governing differential equation for heat conduction through the plate can be written as:

$$
\begin{aligned}
& \frac{\partial \mathrm{T}}{\partial \mathrm{t}}=\mathrm{a} \frac{\partial^{2} \mathrm{~T}}{\partial \mathrm{y}^{2}}(0<y<\delta, \tau>0) \\
& \frac{\partial \mathrm{T}}{\partial \mathrm{t}}=\mathrm{a} \frac{\partial^{2} \mathrm{~T}}{\partial \mathrm{y}^{2}}(0<y<\delta, \tau>0)
\end{aligned}
$$

Where $\mathrm{a}=\mathrm{k} / \mathrm{\rho} \mathrm{C}_{\mathrm{p}}$

Initial condition is a uniform temperature

$$
\mathrm{T}(\mathrm{y}, 0)=\mathrm{T}_{0}(0 \leq \mathrm{y} \leq \delta)
$$

Boundary condition

$$
\begin{gathered}
\left.\frac{\partial \mathrm{T}(\mathrm{y}, \mathrm{t})}{\partial \mathrm{y}}\right|_{\mathrm{y}=0}=0 \\
\left.\mathrm{k} \frac{\partial \mathrm{T}(\mathrm{y}, \mathrm{t})}{\partial \mathrm{y}}\right|_{\mathrm{y}=8}=\mathrm{h}\left[\mathrm{T}_{\mathrm{w}}(\mathrm{s}, \mathrm{t})-\mathrm{T}_{\mathrm{r}}\right]
\end{gathered}
$$

The mathematical problem defined by Eqs. 1 through 4 can be completely nondimensionalized.

Non-dimensional variables can be written as follows:

$$
\eta=\frac{y}{\delta}
$$




$$
\begin{gathered}
\tau=\frac{\mathrm{t}}{\mathrm{t}_{\mathrm{D}}} \\
\theta=\frac{\mathrm{T}-\mathrm{T}_{\mathrm{r}}}{\mathrm{T}_{\mathrm{O}}-\mathrm{T}_{\mathrm{r}}}
\end{gathered}
$$

Two non-dimensional parameters, Fourier number $\mathrm{F}_{0}$ and Biot number $\mathrm{Bi}$ are introduced here, Two parameters can be defined as

$$
\begin{aligned}
& F_{0}=\frac{a t_{D}}{\delta^{2}} \\
& B_{i}=\frac{h \delta}{k}
\end{aligned}
$$

In terms of these non-dimensional variables, the simplified thermal problem can be written in the following non-dimensional form

$$
\frac{\partial \theta}{\partial \tau}=F_{0}\left(\frac{\partial^{2} \theta}{\partial \eta^{2}}\right)
$$

The boundary condition at $\eta=1$ becomes

$$
\frac{\partial \theta}{\partial \eta}=B_{i}
$$

The boundary condition at $\eta=0$ becomes

$$
\frac{\partial \theta}{\partial \eta}=0
$$

The initial condition becomes

$$
\theta=1
$$

An analytical solution can be written as follows

$$
\begin{gathered}
\theta(\eta, \tau)=\sum_{n=0}^{\infty} C_{n} \exp \left(-\mu_{n}^{2} F_{0} \tau\right) \cos \left(\mu_{n} \eta\right) \\
C_{n}=\frac{2 \sin \mu_{n}}{\mu_{n}+\cos \mu_{n} \sin \mu_{n}} \\
\tan \mu_{n}=\frac{B i}{\mu_{n}}
\end{gathered}
$$

The analytical solution can be simplified as

$$
\theta(\tau, \eta)=\theta\left(\mathrm{F}_{0,} \mathrm{Bi}\right)
$$

From Eqs, 15, it has been shown that non-dimensional thermal response is completely governed by two non-dimensional parameters, $\mathrm{F}_{0}$ and $\mathrm{Bi}$.

However, the value of physical temperature dependents on seven physical parameters, physical parameters of materials, material size, heating time, convection coefficient and adiabatic temperature, physical temperature can be written as 


$$
\mathrm{T}(\mathrm{y}, \mathrm{t})=\mathrm{T}\left(\mathrm{p}, \mathrm{k}, \mathrm{Cp}, \mathrm{t}_{\mathrm{D}}, \delta, \mathrm{h}, \mathrm{T}_{\mathrm{r}}\right)
$$

Comparing (15) with (16), it has been shown that the dependence of non-dimensional thermal response on relevant material parameters has been reduced from 7 to only 2 after nondimensionalization.

In our paper, two non-dimensional parameters have been introduced to decide the nondimensional thermal response. These two variables account for the thermal material properties ( $\mathrm{p}$, $\mathrm{k}, \mathrm{Cp}$ ) as well as for the geometric parameters of structure as shown in (8) and (9). In addition, Biot number also combines thermal material properties with thermal environment parameters.

If we change parameters in (8) and (9) according to table 1 , it can be found that $\mathrm{F}_{\mathrm{O}} \mathrm{Bi}$ still maintain unchangeable, the temperature distribution remains unchanged. Therefore, through the adjustment of physical parameters, thermal response can be designed according to our requirements.

Table 1. Value of physical parameters

\begin{tabular}{|c|c|c|c|}
\hline state & 1 & 2 & 3 \\
\hline Convection efficient & $\mathrm{h}$ & $2 \mathrm{~h}$ & $2 \mathrm{~h}$ \\
\hline conductivity & $\mathrm{k}$ & $2 \mathrm{k}$ & $\sqrt{2} k$ \\
\hline density & $\mathrm{p}$ & $\sqrt{2} \mathrm{p}$ & $2 \mathrm{p}$ \\
\hline Heat capacity & $C_{\mathrm{p}}$ & $\sqrt{2} C_{p}$ & $\sqrt{2} C_{p}$ \\
\hline Material size & $\delta$ & $\delta$ & $1 / \sqrt{2} \delta$ \\
\hline \multicolumn{4}{|l}{} \\
\hline$E_{0}$ & $E_{0}$ & $E_{0}$ & $F_{0}$ \\
\hline$B_{\tilde{i}}$ & $B_{\tilde{i}}$ & $B_{\tilde{i}}$ & $B_{\tilde{i}}$ \\
\hline Temperature & $\mathrm{T}$ & $\mathrm{T}$ & $\mathrm{T}$ \\
\hline
\end{tabular}

When Mach number reaches 25 during hypersonic vehicles re-entry, the temperature will be up to $1600 \mathrm{~K}$, and chemical energy and wall radiation must be considered. The net heat flux of heating surface can be written as

$$
\begin{gathered}
q_{n}=q_{o r}\left(1-\frac{h_{w}}{h_{r}}\right)-q_{r a d} \\
q_{r a d}=\varepsilon \sigma T_{w}^{4} \\
h_{w}=f\left(P_{v} T_{w}\right)
\end{gathered}
$$

Where qor denotes cold wall heat flux, $\mathrm{h}_{\mathrm{w}}$ is wall enthalpy, as function of pressure and wall temperature, $\mathrm{h}_{\mathrm{r}}$ is adiabatic wall enthalpy.

It should be noted that wall radiation heat flux in Eqs.17 is proportional to temperature to the fourth power. It is difficult to obtain analytical solution to the heat conduction equation. Therefore, numerical calculation of thermal response has been performed. Moreover, comparison between analytical results and numerical results of thermal response has been done.

\section{Results and discussion}

Consider one dimensional transient heat analysis of infinite plate,

Thickness of plate: $\delta=0.04 \mathrm{~cm}$

Physical properties of high conductivity $\mathrm{C} / \mathrm{C}$ materials: 


$$
\begin{aligned}
\lambda_{1} & =70.0 \mathrm{~W} / \mathrm{m} \cdot \mathrm{K} \\
\mathrm{C}_{\mathrm{P}_{1}} & =1230 \mathrm{~J} / \mathrm{Kg} \cdot \mathrm{K} \\
\mathrm{P}_{1} & =1800 \mathrm{Kg} / \mathrm{m}^{\mathrm{a}}
\end{aligned}
$$

Initial temperature: $\mathrm{T}_{0}=300 \mathrm{~K}$

Boundary conditions:

$$
\begin{gathered}
\left.\frac{\partial t(y, \tau)}{\partial y}\right|_{y=0}=0 \\
\left.\lambda \frac{\partial t(y, \tau)}{\partial y}\right|_{y=8}=q_{o r}\left(1-\frac{h_{w}}{h_{r}}\right)-\varepsilon \sigma T_{w}{ }^{4}
\end{gathered}
$$

\begin{tabular}{|c|c|c|c|c|c|c|c|c|c|c|}
\hline state & $\begin{array}{c}H_{r} \\
(\mathrm{KJ} / \mathrm{K} \\
\mathrm{g})\end{array}$ & $\begin{array}{c}q_{o r} \\
(\mathbf{K W} / \mathbf{m} \\
2\end{array}$ & $q_{r}$ & $\begin{array}{l}\mathbf{K}_{2} / \\
\mathbf{K}_{1}\end{array}$ & $P_{2} / P_{1}$ & $c_{p 2} / c$ & $\delta_{2} / \delta_{1}$ & $T_{W}(\mathrm{~K})$ & $T_{\tilde{b}}(\mathrm{~K})$ & $\begin{array}{c}\text { Heating } \\
\text { time(s) }\end{array}$ \\
\hline 1 & 4000 & 4000 & Yes & 1 & 1 & 1 & 1 & 2330.0 & 2329.9 & $400 \mathrm{~s}$ \\
\hline 2 & 4000 & 4000 & Yes & 2 & $\sqrt{2}$ & $\sqrt{2}$ & 1 & 2323.9 & 2320.3 & $400 \mathrm{~s}$ \\
\hline 3 & 4000 & 4000 & Yes & $\sqrt{2}$ & 2 & $\sqrt{2}$ & $1 / \sqrt{2}$ & 2323.9 & 2320.3 & $400 \mathrm{~s}$ \\
\hline 4 & 4000 & 2000 & No & 1 & 1 & 1 & 1 & 2971.9 & 2955.5 & $400 \mathrm{~s}$ \\
\hline 5 & 4000 & 4000 & No & 2 & $\sqrt{2}$ & $\sqrt{2}$ & 1 & 2971.9 & 2955.6 & $400 \mathrm{~s}$ \\
\hline 6 & 4000 & 4000 & No & $\sqrt{2}$ & 2 & $\sqrt{2}$ & $1 / \sqrt{2}$ & 2971.9 & 2955.5 & $400 \mathrm{~s}$ \\
\hline 7 & 4000 & 2000 & No & 1 & 1 & 1 & 1 & 2064.7 & 2063.4 & $400 \mathrm{~s}$ \\
\hline
\end{tabular}

Thermal environment parameters, material properties and results are listed in table 2.

Table 2. Value of thermal environment parameters, material properties and results

Effects of thermal environment parameters $\left(\mathrm{H}_{\mathrm{r}}, \mathrm{q}_{\mathrm{or}}\right)$, radiation, physical parameters of materials $(p, k, C p)$ and plate size $(\delta)$ on thermal response prediction of surface temperature and back temperature are compared. It should be noticed that $\mathrm{F}_{0}, \mathrm{Bi}$ maintain invariance.

Effects of physical parameters of materials and plate size (state 1,2,3 listed in table 2) on thermal response are shown in Fig.1. Under same thermal environment, just change physical parameters of materials and plate size, finally radiation equilibrium temperature reach to the same value, but thermal response are different due to difference of parameters concerned. Therefore, it can be concluded that it is necessary to delay radiation equilibrium time, increase the materials temperature capability during the thermal protection design.

Effects of thermal environment, physical parameters and plate size (state 4, 5, 6 shown in table 2) on thermal response are shown in Fig.2. If wall radiation heat flux is not neglect and total heat flux is doubled, physical parameters of materials and plate size may have many mapping modes while keeping $\mathrm{F}_{\mathbb{Q}}, \mathrm{Bi}$ equal, thermal response of surface and back will remain unchanged. 


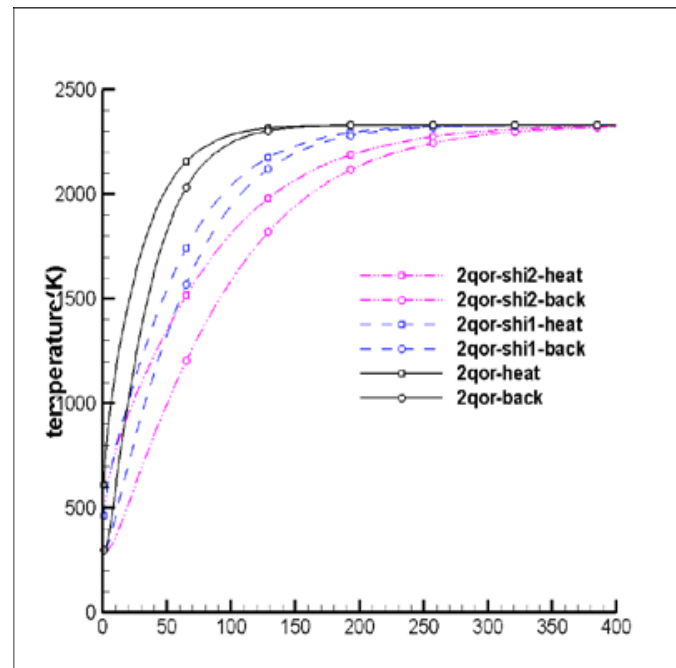

Figure 1. Effects of physical parameters and plate size on thermal response

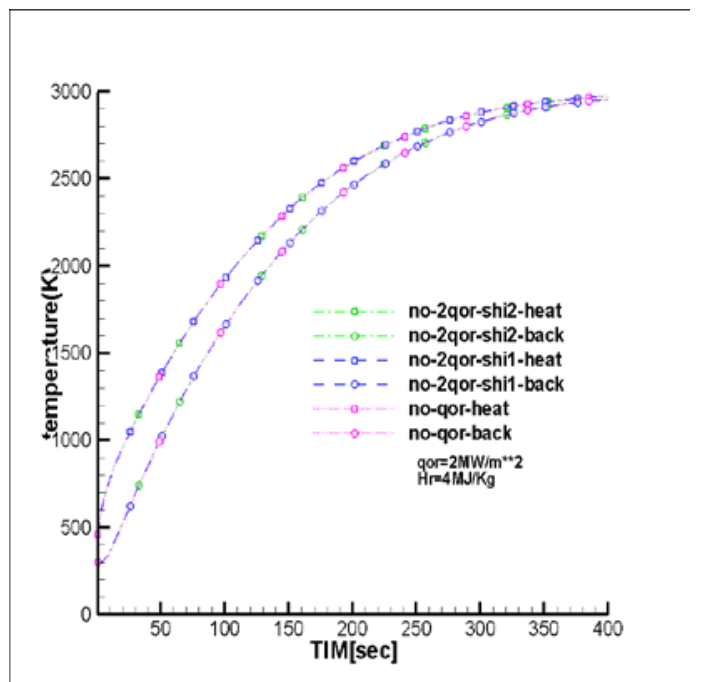

Figure 2. Effects of thermal environment, physical parameters and plate size on thermal response

Fig. 3 illustrates the effects of wall radiation (state 4 and 7 listed in table 2) on the surface. In the figure, it has been shown that effects of wall radiation may be neglect when surface temperature is less than $1200 \mathrm{~K}$. Wall radiation leads to a sharp decrease of surface temperature and have shorten radiation equilibrium time when surface temperature reaches up to $1200 \mathrm{~K}$. Surface temperature has been decreased by $900 \mathrm{~K}$ after 400 s heating.

Effects of physical parameters of materials on thermal response (state 1, 4, 5 )are shown in Fig.4. During the early heating time, before wall temperature reaches $1200 \mathrm{~K}$, if heat flux is doubled, through adjusting physical parameters of materials, temperature distributions could remain unchangeable. But when surface temperature reaches up to about $1200 \mathrm{~K}$ (back temperature to about $1300 \mathrm{~K}$ ), variations of physical parameter of materials take little effect on thermal response, Effects of thermal environment parameters $\left(\mathrm{H}_{\mathrm{T}}, \mathrm{q}_{\mathrm{or}}\right)$ become more and more important.

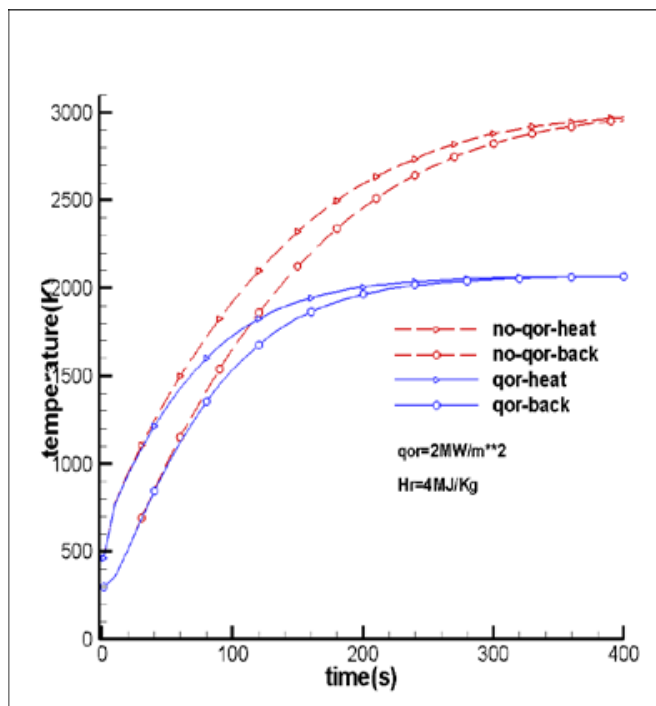

Figure 3. Effects of radiation on thermal response

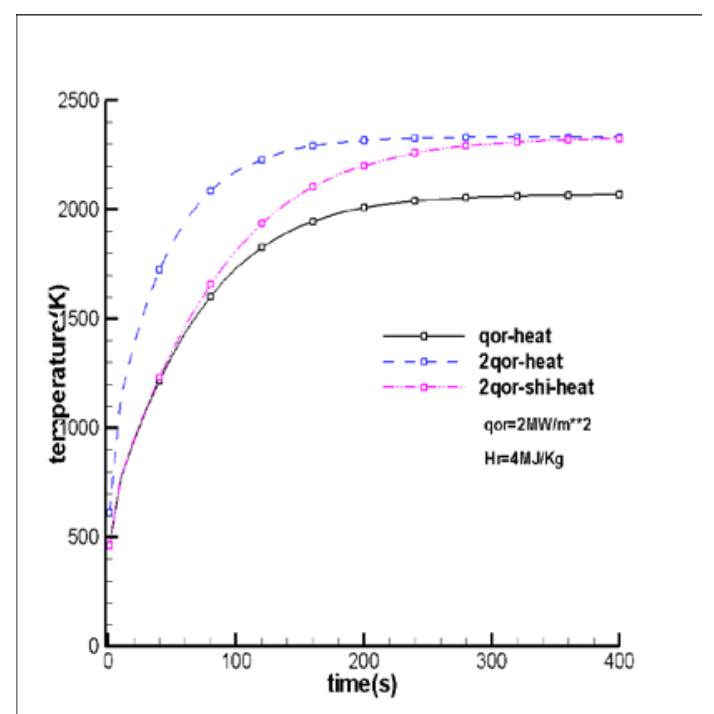

Figure 4. Effects of physical parameters on thermal response of surface 


\section{Conclusion}

A simplified transient thermal problem was investigated in an attempt to gain basic insight into thermal response of non-ablation materials subjected to a transient aerodynamic heating plus.

An analytical solution was derived for the transient response of this simplified transient problem. The analytical solution introduces two non-dimensional parameters. A numerical study was also performed to obtain thermal response of materials. Furthermore, Analysis of influencing factors on transient thermal response has been performed.

Results and data obtained from calculation will give guidance to thermal protection design.

\section{References}

1. Christian Gogu, Raphael T. Haftka, Satish K. Bapanapalli, and Bhavani V. Sankar,

2. Dimensionality Reduction Approach for Response Surface Approximations: Application to Thermal Design,AIAA JOURNAL, Vol. 47, No. 7, July 2009

3. Max L. Blosser,Analysis and Sizing for Transient Thermal Heating of Insulated Aerospace Vehicle Structures, NASA/TP-2012-217595

4. Jiang gui-qing, non-ablation thermal protection system and mechanism, Jan, 2005 